\title{
Prediction of Consistency of Pituitary Adenomas by Magnetic Resonance Imaging
}

\author{
Kyle A. Smith ${ }^{1}$ John D. Leever ${ }^{2}$ Roukoz B. Chamoun ${ }^{1}$ \\ 1 Department of Neurosurgery, University of Kansas Medical Center, \\ Kansas City, Kansas, United States \\ 2 Department of Radiology, University of Kansas Medical Center, \\ Kansas City, Kansas, United States
}

Address for correspondence Roukoz B. Chamoun, MD, Department of Neurosurgery, University of Kansas Medical Center, 3901 Rainbow Blvd., 3021, Kansas City, KS 66160, United States (e-mail: rchamoun@kumc.edu).

J Neurol Surg B 2015;76:340-343.

\begin{abstract}
Keywords

- pituitary adenomas

- fibrous adenomas

- adenoma consistency

- endoscopic transsphenoidal

- MRI

Objective Pituitary adenomas are typically soft. The prevalence of fibrous adenomas is $\sim 5$ to $13 \%$. Firm tumors are difficult to remove by curettage or suction. Predicting fibrous adenomas by magnetic resonance (MR) imaging is typically difficult and unreliable. We propose a new prediction method based on MR T2-sequence intensity. Methods The MRIs of 36 consecutive patients with nonsecreting macroadenomas were evaluated preoperatively by a blinded radiologist. Using an MR T2-weighted sequence, regions of interest were sampled from the adenoma and cerebellar peduncle, and the ratio was calculated. Intraoperatively, tumors were graded prospectively for their consistency by the operating surgeon.

Results There were 28 soft and 6 fibrous tumors. Unpaired $t$ test for these ratios was found to be statistically significant ( $p<0.0240$; $95 \%$ confidence interval, -0.8229 to -0.06207 ). Mean values for soft tumors were found to be 1.918 (standard error of the mean $[\mathrm{SEM}]=0.08212$ ); firm tumors, 1.475 (SEM $=0.1179$ ). Soft tumors were associated with ratios $>1.5$ (sensitivity $100 \%$; specificity $66.7 \%$ ); firm tumors were associated with ratios $<1.8$ (sensitivity $100 \%$; specificity $42.9 \%$ ).

Conclusion Fibrous adenomas are typically diagnosed intraoperatively. However, their resection can be technically challenging. Using $T 2$ intensity ratios on routine preoperative MRI allows identification of these challenging cases. The surgeon can then be better prepared for the surgical resection.
\end{abstract}

\section{Introduction}

Pituitary adenomas are overwhelmingly of a soft consistency, so typical methods of resection with curettage and suction are adequate. ${ }^{1-4}$ The utility of the endonasal transsphenoidal approach has made it the standard of care for most surgeons. Adenomas can present a surprisingly firm consistency, however, $\sim 5$ to $13 \%$ of the time. ${ }^{1-5}$ In these cases tumor consistency may pose a real challenge to resection via the transsphenoidal approach. ${ }^{5}$ Knowledge of the consistency preoperatively may assist the surgeon with surgical planning or the referral to a tertiary center for these difficult lesions.

received

December 30, 2014

accepted

February 2, 2015

published online

April 27, 2015
Based on the available literature, no definitive conclusion exists for the correlation of adenoma consistency with radiologic imaging. One study demonstrates that a lower signal on magnetic resonance (MR) T2-weighted imaging correlates with increased collagen content at pathologic diagnosis and therefore a fibrous consistency. ${ }^{2}$ Another article concluded that diffusionweighted imaging intensity values correspond with the percentage of collagen content on pathologic examination. ${ }^{4}$ Other articles conclude that no statistically significant correlation could be found between consistency and imaging. ${ }^{1,3,5-7}$ White matter was used for signal intensity comparison in two studies. (c) 2015 Georg Thieme Verlag KG Stuttgart · New York
DOI http://dx.doi.org/ 10.1055/s-0035-1549005. ISSN 2193-6331. 
We hypothesized that ratios of signal intensity of adenoma to cerebellar peduncle on MR T2-weighted imaging would allow us to predict consistency while reliably standardizing MR signals to a consistent anatomical landmark. T2-weighted images should reflect the water content of the tumor and thus its consistency. We used the cerebellar peduncle as an internal reference for T2 intensity. The cerebellar peduncle is a large anatomical landmark that allows easy reproducibility.

\section{Methods}

\section{Patients}

Thirty-six consecutive patients who underwent resection of nonsecreting macroadenomas at one academic medical center were included in this imaging study. Institutional review board approval was obtained.

\section{Clinical Setting}

All patients were operated on by a single surgeon (R. C.) with cooperation for approach and closure with the otolaryngology service at this institution. During surgery, the consistency category (soft or firm) was applied prospectively by the primary neurosurgeon. Adenomas that were easily removed in the standard fashion with curettage and suction were designated as soft tumors. Adenomas that were resistant to removal by curettage or required adjuvant methods were designated as firm tumors. Even though this definition is rather subjective, it is actually widely used and does make sense clinically to the operating surgeon.

\section{Imaging}

In choosing an imaging modality, a standard imaging sequence was chosen for developing a method for consistency prediction. The use of a standard imaging modality and sequence thus allows for further application without the need for special radiologic equipment or selective training for radiologists.

The MR images of these patients were reviewed independently by a blinded radiologist (J. L.). Utilizing the MR T2-weighted sequence, a ratio was calculated with adenoma to cerebellar peduncle measurements ( - Fig. 1). An example of these ratio calculations are observed in $-\mathbf{F i g}$. 2. A region of interest (ROI) was selected within the adenoma for a representative sampling in homogenous intensity. Alternatively, multiple ROIs were selected for mixed intensity lesions, and an average intensity value was calculated for the lesion. A ROI was chosen from the cerebellar peduncle. This peduncle ROI was a uniform shape and size (circular) and selected by the blinded radiologist (J. L.). By utilizing a standard anatomical denominator and one that is easily selected, we believe sampling error is reduced and MR signals are standardized to the individual MR scan. This process serves as a method of internal control and allows quantitative comparison of various MR scans. Using MR T2-weighted sequence ROIs, adenoma-to-cerebellar peduncle ratios were calculated independently of intraoperative consistency. Ratios and consistency categories, namely firm or soft, were then analyzed using an unpaired $t$ test.

\section{Results}

Imaging and operative consistency were collected on 36 consecutive patients. Raw patient data are shown in -Table 1. Two patients could not have imaging ratios calculated due to inadequate outside hospital scans. Imaging ratios were available in 34 patients. Fifteen patients were male; 19 were female. Patient age ranged from 29 to 85 years; mean age was 54.53 years.

Intraoperative consistency was recorded based on resection characteristics and ease of adenoma removal. Six
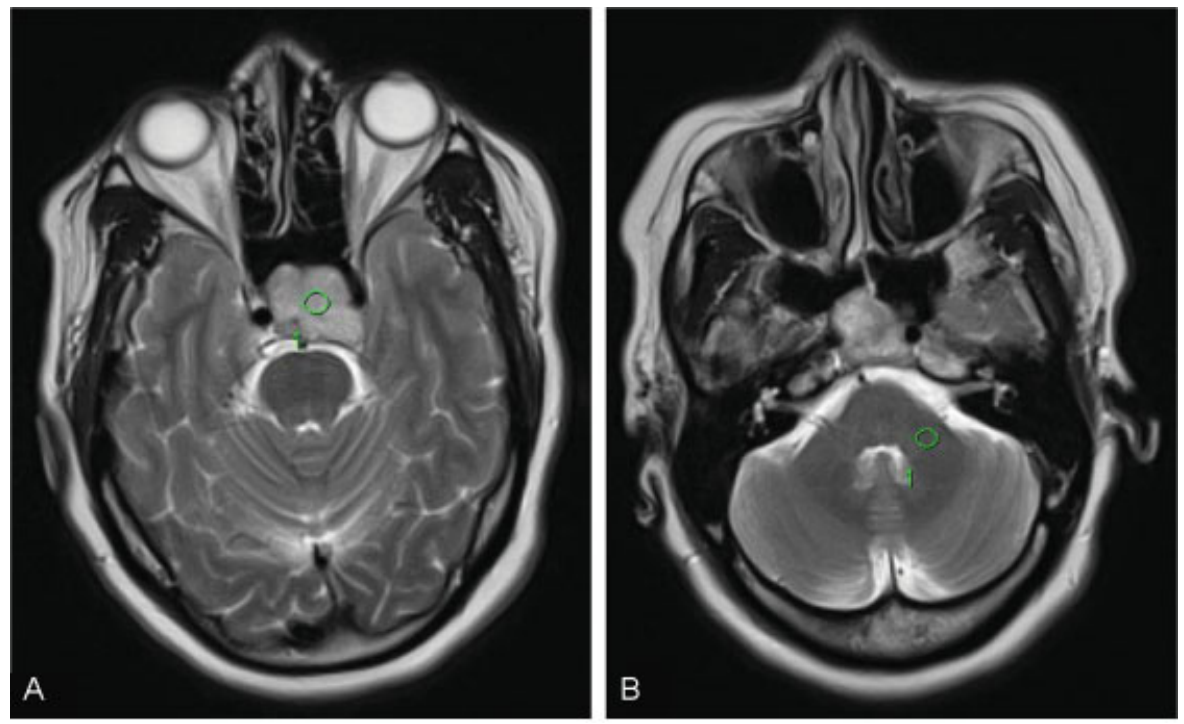

Fig. 1 How to use the adenoma- to-cerebellar peduncle ratio. (A) Region of interest (ROI) in adenoma on T2 sequence. (B) ROI in middle cerebellar peduncle on $\mathrm{T} 2$ sequence. Calculating the adenoma-to-cerebellar peduncle ratio. Ratio = intensity value for ROI within tumor (A)/Intensity value for ROI within middle cerebellar peduncle (B). 


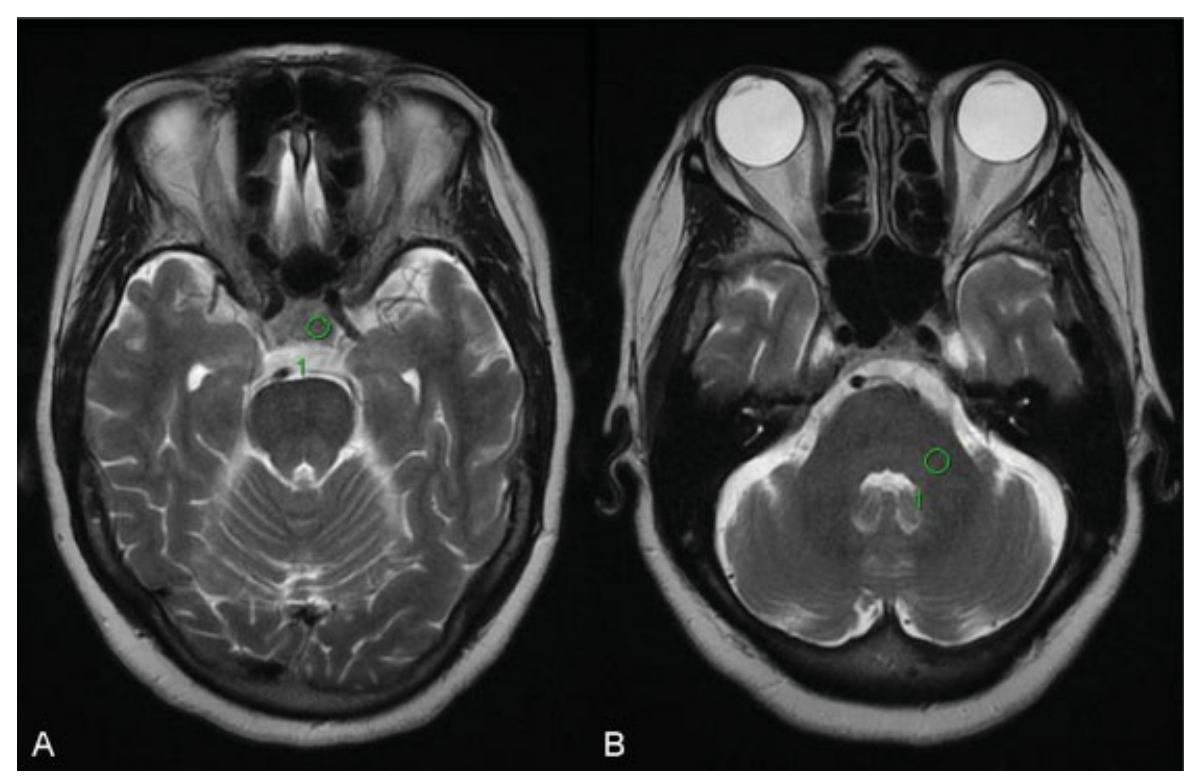

Fig. 2 Adenoma-to- cerebellar peduncle intensity ratio example. (A) Region of interest (ROI) intensity within tumor $=938.4$ (B) ROI intensity within middle cerebellar peduncle $=977.5$. ACP ratio $=938.4 / 977.5=0.96$.

tumors were firm or fibrous; 28 were soft. Frequency distribution of consistency in plotted in - Fig. 3. Overall adenoma-to-cerebellar peduncle ratios ranged from 0.96 to 3.27. Overall mean ratio was 1.84 . Raw data were analyzed via an unpaired $t$ test for ratios compared with clinical consistency based on resection characteristics. Mean values for soft tumors were found to be 1.918 (standard error of the mean $[\mathrm{SEM}]=0.08212$ ); thus the mean range was 1.84 to 2.00. Mean values for firm tumors were found to be 1.475 ( $\mathrm{SEM}=0.1179$ ); thus the mean range was 1.36 to 1.59. Overall $t$ test found the data to be statistically significant $(p<0.0240 ; 95 \%$ confidence interval [CI], -0.8229 to $-0.06207)$. For firm tumor ratios, the $95 \% \mathrm{CI}$ was 1.172 to 1.778. For soft tumor ratios, the $95 \% \mathrm{CI}$ was 1.749 to 2.086 . All soft tumors had ratios $>1.5$ (sensitivity $100 \%$; specificity $66.7 \%$ ). Positive predictive value (PPV) of ratio $>1.5$ for soft tumor was $93.1 \%$. Negative predictive value (NPV) of ratio $>1.5$ was $100 \%$. All firm tumors had ratios $<1.8$

\section{Frequency Distribution for Intensity Ratios}

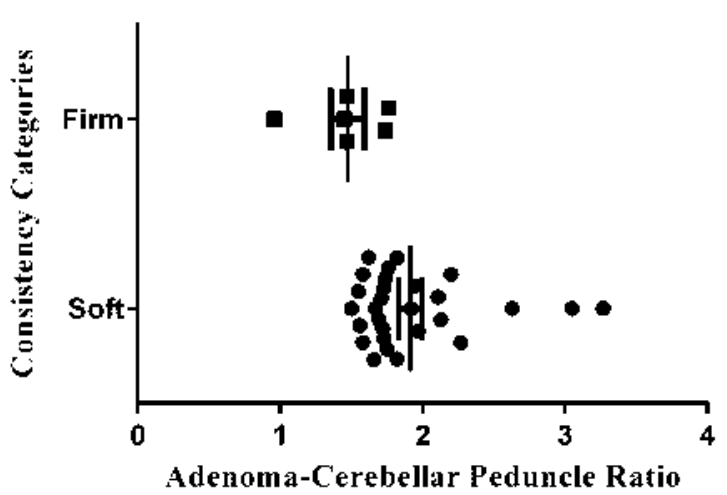

Fig. 3 Frequency distribution for adenoma-cerebellar peduncle intensity ratios by consistency. (sensitivity $100 \%$; specificity $42.9 \%$ ). PPV of ratio $<1.8$ for firm tumors was $27.3 \%$. NPV of ratio $<1.8$ was $100 \%$.

\section{Discussion}

Consistency of pituitary adenomas is an important variable in the surgical resection of these lesions. Prediction of consistency of adenomas, namely soft or firm, carries implications for the neurosurgeon. Fibrous adenomas are often difficult to resect. Prediction of firm adenomas allows planning for surgery such as the use of extracapsular dissection and the availability of surgical adjuncts such as a side-cutting instrument like the NICO myriad in our series (NICO Corp., Indianapolis, Indiana, United States). Patient care and safety may be affected as well if fibrous adenomas are able to be predicted and referred to tertiary centers, if needed.

In our study we standardized inter-MRI signal intensity by utilizing a control of cerebellar peduncle consistency within the same patient study. Additionally, we attempted to standardize the ratio and selection bias among users by choosing this sizable consistent anatomical feature. Although controversy exists as to whether MRI correlates with consistency and the ideal sequence, we chose to reinvestigate $\mathrm{T} 2$-weighted sequence due to its versatility. Mean values of either group reached statistical significance. Utilizing SEM, we approximated ratio cutoff values for these categorical extremes (firm or soft). None of our fibrous tumors had ratios $>1.8$; none of our soft tumors had ratios $<1.5$. Tumors with ratios between 1.5 and 1.8 can be either soft or fibrous. These ratio cutoff values serve as good screening tools with high sensitivity and good NPV for identification of tumors that are likely to be firm.

Limitations of our study do exist. First, our series consisted of a mere 36 patients, which is a small series. Our consistency categories were dichotomized into just two groups, but in 
Table 1 Patient data

\begin{tabular}{|c|c|c|c|c|}
\hline S. No. & Age, y & Sex & ACP ratio & Clinical consistency \\
\hline 1 & 62 & $\mathrm{~F}$ & 1.73 & Soft \\
\hline 2 & 49 & $\mathrm{~F}$ & 1.76 & Fibrous \\
\hline 3 & 33 & $\mathrm{~F}$ & 1.76 & Soft \\
\hline 4 & 48 & $\mathrm{~F}$ & 1.95 & Soft \\
\hline 5 & 57 & $\mathrm{~F}$ & 1.56 & Soft \\
\hline 6 & 52 & $\mathrm{~F}$ & 1.92 & Soft \\
\hline 7 & 70 & $\mathrm{M}$ & 2.13 & Soft \\
\hline 8 & 66 & M & 1.74 & Fibrous \\
\hline 9 & 61 & $\mathrm{M}$ & 1.73 & Soft \\
\hline 10 & 54 & $\mathrm{M}$ & 1.58 & Soft \\
\hline 11 & 68 & $\mathrm{~F}$ & 1.62 & Soft \\
\hline 12 & 52 & $M$ & 2.11 & Soft \\
\hline 13 & 47 & $\mathrm{~F}$ & 1.66 & Soft \\
\hline 14 & 37 & M & 1.50 & Soft \\
\hline 15 & 70 & $\mathrm{M}$ & 2.27 & Soft \\
\hline 16 & 85 & $\mathrm{~F}$ & 1.47 & Fibrous \\
\hline 17 & 29 & $\mathrm{~F}$ & 1.67 & Soft \\
\hline 18 & 49 & $\mathrm{M}$ & 1.75 & Soft \\
\hline 19 & 72 & $\mathrm{M}$ & 1.74 & Soft \\
\hline 20 & 44 & $M$ & 1.82 & Soft \\
\hline 21 & 48 & $\mathrm{~F}$ & 1.97 & Soft \\
\hline 22 & 35 & $\mathrm{M}$ & 1.55 & Soft \\
\hline 23 & 66 & $\mathrm{M}$ & 1.82 & Soft \\
\hline 24 & 69 & $\mathrm{~F}$ & 1.71 & Soft \\
\hline 25 & 72 & $\mathrm{~F}$ & 1.58 & Soft \\
\hline 26 & 57 & $\mathrm{~F}$ & 2.20 & Soft \\
\hline 27 & 45 & $\mathrm{M}$ & 1.45 & Fibrous \\
\hline 28 & 39 & $\mathrm{~F}$ & 1.69 & Soft \\
\hline 29 & 69 & $\mathrm{M}$ & NA & Fibrous \\
\hline 30 & 34 & $\mathrm{~F}$ & 3.27 & Soft \\
\hline 31 & 42 & $\mathrm{M}$ & 2.63 & Soft \\
\hline 32 & 56 & $\mathrm{M}$ & NA & Soft \\
\hline 33 & 78 & $\mathrm{~F}$ & 0.96 & Fibrous \\
\hline 34 & 67 & $\mathrm{M}$ & 1.47 & Fibrous \\
\hline 35 & 50 & $\mathrm{~F}$ & 1.72 & Soft \\
\hline 36 & 47 & $\mathrm{~F}$ & 3.05 & Soft \\
\hline
\end{tabular}

Abbreviations: F, female; M, male; NA, not available; ACP, adenomacerebellar peduncle. clinical practice a continuum of consistency exists. Therefore overlap of signal ratios occurred but likely represents those adenomas of an intermediate group of characteristics of either group. With our ratio values selected, we aim to select those adenomas of either extreme because of their clinical significance.

\section{Conclusion}

Adenoma-to-cerebellar peduncle ratios serve as good screening tools for adenomas that may be firm in consistency. Ratios $>1.8$ have a high predictive value for soft consistency adenomas; ratios $<1.5$ has a high predictive value for firm consistency adenomas.

Notes

This article was scheduled for oral presentation at the North American Skull Base Society February 2015 conference.

\section{Disclaimer}

The authors report no conflict of interest concerning the materials or methods used in this study or the findings specified in this article.

\section{References}

1 Bahuleyan B, Raghuram L, Rajshekhar V, Chacko AG. To assess the ability of MRI to predict consistency of pituitary macroadenomas. Br J Neurosurg 2006;20(5):324-326

2 Iuchi T, Saeki N, Tanaka M, Sunami K, Yamaura A. MRI prediction of fibrous pituitary adenomas. Acta Neurochir (Wien) 1998;140(8): 779-786

3 Mahmoud OM, Tominaga A, Amatya VJ, et al. Role of PROPELLER diffusion-weighted imaging and apparent diffusion coefficient in the evaluation of pituitary adenomas. Eur J Radiol 2011;80(2):412-417

4 Pierallini A, Caramia F, Falcone C, et al. Pituitary macroadenomas: preoperative evaluation of consistency with diffusionweighted MR imaging-initial experience. Radiology 2006; 239(1):223-231

5 Naganuma H, Satoh E, Nukui H. Technical considerations of transsphenoidal removal of fibrous pituitary adenomas and evaluation of collagen content and subtype in the adenomas. Neurol Med Chir (Tokyo) 2002;42(5):202-212; discussion 213

6 Chakrabortty S, Oi S, Yamaguchi M, Tamaki N, Matsumoto S. Growth hormone-producing pituitary adenomas: MR characteristics and pre- and postoperative evaluation. Neurol Med Chir (Tokyo) 1993;33(2):81-85

7 Hagiwara A, Inoue Y, Wakasa K, Haba T, Tashiro T, Miyamoto T. Comparison of growth hormone-producing and non-growth hormone-producing pituitary adenomas: imaging characteristics and pathologic correlation. Radiology 2003;228(2):533-538 